\title{
The Barcode of Life Initiative: Reply to Dupré, Hollingsworth and Holm
}

\author{
FILIPE O. COSTA AND GARY R. CARVALHO ${ }^{1}$
}

We are grateful to John Dupré, Peter Hollingsworth and Petter Holm for their insightful and constructive responses to our article. ${ }^{2}$ As with any new and increasingly applied approach, DNA barcoding has provoked considerable discussion, even though the basic technology employed is essentially a refinement of existing molecular approaches to systematics. ${ }^{3}$ What characterises DNA barcoding is the attempt to standardise the molecular approach by focusing on one or a few genes with appropriate levels of among-species divergence, and to secure global accessibility to a common database. Additionally, although one gene, cytochrome oxidase I (COI), has proven to be informative across diverse taxa, the aim of DNA barcoding has not been to identify a single common gene, but rather to maximise standardisation across related taxa to ensure high comparability. DNA barcoding is essentially a practical tool that can be applied to compare a target DNA sequence with a reference DNA sequence that may confirm species identity or generate alternative hypotheses of species delineation. It is crucial therefore to appreciate that rather than replacing conventional approaches to taxonomy, which rely heavily on ecological, morphological and behavioural characteristics, DNA barcoding can in many cases render the Linnaean system more accessible. A recent cover of Nature $e^{4}$ illustrating a modern-age Linnaeus wearing a contemporary naturalist's outfit and holding a barcode in his hand could not be more paradigmatic.

Rather than rehearse many previous discussions and articles on the merits and limitations of DNA barcoding, here we focus on just a few of the key points raised by Dupré, Hollingsworth and Holm. One of the initial points raised by John Dupré is the relative bias of existing DNA barcoding databases towards eukaryotes, especially animals. While the balance of current information is indeed skewed toward animals, the utilisation of alternative standardised gene sequences is being increasingly used in other groups, including land plants, ${ }^{5}$ fungi ${ }^{6,7}$ and other protists. ${ }^{8}$ The driving characteristic of such inventories of biodiversity is to ensure high comparability and quality of reference databases. While it is clear, as with any technology, that certain taxa may remain recalcitrant to standard barcoding approaches, occasional combination of additional sequences, might be anticipated to disclose species identity for many organisms.

A major point made by Dupré is the link between DNA barcoding and the biological species concept. Although DNA barcoding may provide novel insights into the species concept,${ }^{9}$ it is certainly not the primary aim. While there may be direct concordance between presumed species status and reproductive isolation, ${ }^{10}$ barcoding is potentially a practical tool that may facilitate the classification of ecological or morphological diversity within a taxonomic framework. It is not disputed that closely related species experiencing intermittent or frequent hybridisation will not be detected using conventional DNA barcoding approaches. However, where there appears to be 
an uncoupling between observed ecological, behavioural or morphological heterogeneity and reproductive or molecular divergence, DNA barcoding can serve to facilitate the testing of alternative hypotheses or the application of alternative species concepts. Thus, rather than being constrained by or restricted to only those taxa conforming to Mayr's vision of biological species, DNA barcoding can extend taxonomic approaches to test evidence obtained at other biological levels. ${ }^{11}$

A further point raised by Dupré is the necessary limitation of any molecular taxonomy by the availability of high level taxonomic expertise. Coincident with the inclusive biological nature of DNA barcoding, is the recognition that highly trained taxonomists remain a crucial component of the species identification procedure. However, James Hanken, ${ }^{12}$ in an historical overview of the rates of species discovery, suggests that, indeed, taxonomy should rely on technological innovation rather than expecting an improbable substantial enlargement of the community of taxonomic experts. Thus, a more realistic solution would be the implementation of innovative technologies into an integrative taxonomy framework, including digital imaging, high resolution X-rays, information technologies, DNA barcoding and other genomic approaches.

It is expected, however, that barcoding may extend the taxonomic process to those individuals lacking such skills, depending of course on the availability of a matching DNA sequence in the reference database. This point is linked to the more general issue of how DNA barcoding may facilitate interest in taxonomy among the general public, thereby serving to promote a case of conservation measures. It is accepted that many people, including the interested amateur naturalist, are motivated by an innate interest in the nature and patterns of biological diversity that will not necessarily be enhanced by molecular taxonomy. However, non-specialists within conservation bodies, museums and various government laboratories where molecular expertise might not exist can still submit samples to commercial companies for DNA sequencing, enhancing access. Such accessibility will enhance public awareness through the disclosure of new species, as well as increasing the profile of threatened species or risks posed by invasive species. The availability of the so-called 'Tricorder', although a seductive and distinctive vision for the future of DNA barcoding, is only one aspect. The recent discoveries of new species in what are considered welldocumented taxa, such as birds, ${ }^{13,14}$ lepidopterans, ${ }^{15}$ and fish,,${ }^{16}$ enhance the awareness of biodiversity among the general public that may relate more readily to the discovery of new species in easily recognisable and familiar taxa. Such disclosures can then serve as a framework for emphasising the much higher levels of hidden biodiversity and cryptic speciation in less familiar organisms, especially among microbes.

Peter Hollingsworth points out that one of the main drivers for environmental awareness for the general public is likely to remain the day-to-day contacts with biodiversity. While this is undoubtedly true, it is not necessarily exclusively so. As indicated above, increased awareness of environmental issues, which has been driven by such things as climate change and habitat destruction, has focused increasingly on the role of species in ecosystems. Thus, a more precise cataloguing of the levels and distribution of species diversity across the globe can only help to generate a case for public engagement in environmental and conservation policies. 
While Petter Holm promotes many of the virtues of DNA barcoding, he questions the level of investment necessary to generate 'a virtual Linnaeus'. Considerable global effort is already underway with various DNA barcoding campaigns and other biodiversity surveys (eg, Census of Marine Life ${ }^{17}$ ). However, there were two recent and important developments that will have a major impact in accelerating the availability of the 'virtual Linnaeus'. One of them is the International Barcode of Life (iBOL) ${ }^{18}$ an international consortium that aims to generate DNA barcodes for 500,000 species over a period of five years, starting in 2009. While such efforts will of course take time and considerable manpower and funding, they will be rewarded by gains in efficiency - in terms of both time and expenditure - by the scale of activity, the high throughput analysis and automation. It is difficult to envisage how such efficiencies could be generated by the hitherto taxonomic and geographically fragmented efforts to log biodiversity, especially where quality assurance and access to curated voucher specimens is more variable. Another recent salient development, The Encyclopedia of Life (EoL), ${ }^{19}$ brings together the currently scattered global biodiversity initiatives, thereby 'materializing' the virtual Linnaeus. The EoL is conceived as an 'ecosystem of websites that makes all key information about life on Earth accessible to anyone, anywhere in the world'. Ten years is the estimated time for the completion of the species pages for the 1.8 million known species, the first pages are expected to be available sometime in 2008. Inspired by Wikipedia, EoL intends also to consider the contribution of individual citizens, though all published information will be subject to authentication by scientists. DNA barcoding will dovetail well with this project, playing a key role, for instance, in providing unequivocal links between different source databases, such as between museum specimens and genomic databases (eg, GenBank). It is precisely the combined influence of such expansive biodiversity projects that we expect to have a significant impact in the bioliteracy and appeal for biodiversity of future generations.

Holm also raises the ethically important and timely issue of balancing such access and value to DNA barcoding efforts with geographic variability in biodiversity and infrastructure. The Consortium for the Barcode of Life aims to catalogue global biodiversity through the existence of various regional working groups associated with particular taxa. Obtaining and describing such diversity where it is at its greatest in the tropics, for example, but where infrastructure and expertise may be more variable, is a particular challenge. While such issues will serve to constrain overall activity, it is only through the generation of a global effort that sufficient resources and manpower might be mobilised to address such imbalance. The existence of what Holm refers to as 'dark possibilities', whereby DNA barcoding inventories may be exploited by capitalist firms or bio-prospecting excursions, is a possibility where information is available to all. Such activities are of course not new, and although the ethos of DNA barcoding would be counter to such exploitation, scenarios can be envisaged where useful products or species may be disclosed for use not just by the developed world. A case in point is the current DNA barcoding efforts in mosquitoes, ${ }^{20}$ which of course are associated with considerable distribution of disease and mortality. 


\section{Concluding remarks}

It is a useful exercise to critically evaluate the application and implications of new approaches to tackling well established problems such as taxonomy and species identity. DNA barcoding has often been regarded as an alternative or exclusive approach to generate a "new taxonomy". As seen from many published studies on DNA barcoding, it is an approach that is by its nature dependent upon comprehensive reference to other biological levels of organisation. Genes evolve in individuals that often aggregate into populations that live in specific habitats, and it is crucial therefore to examine the extent to which biological heterogeneity may coincide with recognisable species groupings. Where a convenient genetic tag (stable, heritable and discrete) can be developed to recognise such entities, such as a DNA barcode, then this can be a useful practical tool that may, or may not, be used in conjunction with other independent corroboratory information. The integration of molecular approaches with conventional Linnaean taxonomy has in many cases stimulated new levels of investment in taxonomy. ${ }^{21}$ While the prognosis for DNA barcoding appears sound, there will continue to be a need for conventional taxonomic expertise, though one might hope for increased integration and communication across the molecular and non-molecular divide. The key is not to claim exclusivity for DNA barcoding, but rather to promote awareness of the complexity and in some cases the fragility of diversity in the natural biological world.

\footnotetext{
${ }^{1}$ The authors are at the School of Biological Sciences, University of Wales, Bangor, UK. Correspondence to f.o.costa@bangor.ac.uk.

${ }^{2}$ F.O. Costa and G.R. Carvalho. The Barcode of Life Initiative: synopsis and prospective societal impacts of DNA barcoding of fish. Genomics, Society and Policy 2007; 3 (2): 29-40; and commissioned responses in same issue.

${ }^{3}$ A. Ferguson. 1980. Biochemical Systematics and Evolution. Blackie, Glasgow \& London.

${ }^{4}$ Nature 2007; 446 (7133): 231-342, 15 March. Issue dedicated to the celebration of the 300th anniversary of Linnaeus's birth.

${ }^{5}$ W.J. Kress and D.L. Erickson. A two-locus global DNA barcode for land plants: the coding rbcL gene complements the non-coding trnHpsbA spacer region. PLoS One 2007; 6 (e508): 1-10.

${ }^{6}$ X.J.I.A. Min and D.H. Hichey. Assessing the effect of varying sequence length on DNA barcoding of fungi. Molecular Ecology Notes 2007; 7 (3): 365-373.

${ }^{7}$ All Fungi DNA Barcoding Planning Workshop http://barcoding.si.edu/fungi.html.

${ }^{8}$ Report of the Workshop on 'Protistan Barcoding, Reference Material and Cultures', November 6-7, 2006, Portland, ME USA. Protist here are defined as 'mostly microscopic eukaryotic organisms commonly referred to as algae, aquatic fungi, and protozoa'. Available at

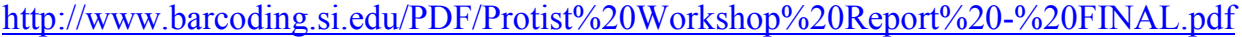

${ }^{9}$ S.E. Miller. DNA barcoding and the renaissance of taxonomy. Proceedings of the National Academy of Sciences 2007; 104 (12): 4775-4776.

${ }^{10}$ A. Gomez et al. Mating trials validate the use of DNA barcoding to reveal cryptic speciation of a marine bryozoan taxon. Proceedings of the Royal Society Series B- 2007; 274 (1607): 199-207.

${ }^{11}$ P.D.N. Hebert et al. Ten species in one: DNA barcoding reveals cryptic species in the neotropical skipper butterfly Astraptes fulgerator. Proceedings of the National Academy of Sciences 2004; 101 (41): 14812-14817.

12 J. Hanken. Taxonomy and Species discovery. DNA Barcoding for CoML Workshop, 15-17 November 2006, The Netherlands Royal Academy of Arts \& Sciences, Amsterdam. Available at http://www.barcoding.si.edu/coml agenda 2006.htm.
} 
${ }^{13}$ P.D.N. Hebert et al. Identification of birds through DNA barcodes. PLoS Biology 2004; 2 (10): 1657-1663.

${ }^{14}$ K.C.R. Kerr et al. Comprehensive DNA barcode coverage of North American birds. Molecular Ecology Notes 2007; 7 (4): 535-543.

${ }^{15}$ Hebert et al., op. cit. note 11.

${ }^{16}$ B.C. Victor. Coryphopterus kuna, a new goby (Perciformes: Gobiidae: Gobiinae) from the western Caribbean, with the identification of the late larval stage and an estimate of the pelagic larval duration. Zootaxa 2007; 1526: 51-61.

${ }^{17}$ Census of Marine Life (CoML): http://www.comlsecretariat.org/

${ }^{18}$ The International Barcode of Life (iBOL) http://www.dnabarcoding.org/

${ }^{19}$ The Encyclopedia of Life (EoL) www.eol.org

${ }^{20}$ N. Kumar Pradeep et al. DNA Barcodes can distinguish species of Indian mosquitoes (Diptera: Culicidae). Journal of Medical Entomology 2007; 44 (1): 1-7.

${ }^{21}$ Miller, op. cit. note 9. 